\title{
The hybrid installation TAIGA: design, status and preliminary results
}

N. Lubsandorzhiev ${ }^{*}, 1$, I. Astapov ${ }^{9}$, P. Bezyazeekov ${ }^{2}$, A. Borodin ${ }^{10}$, M. Brueckner ${ }^{8}$, N. Budnev' ${ }^{2}$ V. Chernykh², A. Chiavassa ${ }^{4}$, A. Dyachok ${ }^{2}$, O. Fedorov², A. Gafarov², A. Garmash ${ }^{11}$, V. Grebenyuk ${ }^{10,14}$, O. Gress ${ }^{2}$, T. Gress ${ }^{2}$, A. Grinyuk ${ }^{10}$, O. Grishin ${ }^{2}$, D. Horns ${ }^{6}$, A. Igoshin ${ }^{1}$, A. Ivanova ${ }^{2}$, N. Kalmykov' ${ }^{1}$ Y. Kazarina' ${ }^{2}$, V. Kindin' ${ }^{9}$, P. Kirilenko ${ }^{11}$, S. Kiryuhin ${ }^{2}$, R. Kokoulin ${ }^{9}$, K. Kompaniets ${ }^{9}$, E. Korosteleva ${ }^{1}$, V. Kozhin ${ }^{1}$, E. Kravchenko ${ }^{11,12}$, A. Kryukov ${ }^{1}$, L. Kuzmichev 1,2, A. Lagutin ${ }^{15}$, Y. Lemeshev ${ }^{2}$, V. Lenok ${ }^{2}$, B. Lubsandorzhiev ${ }^{3,1}$, R. Mirgazov' ${ }^{2}$, R. Mirzoyan ${ }^{5,1}$, R. Monkhoev' ${ }^{2}$, E. Osipova ${ }^{2}$, A. Pakhorukov², A. Pan ${ }^{10}$, M. Panasyuk ${ }^{1}$, L. Pankov' ${ }^{2}$, A. Petrukhin ${ }^{9}$, V. Poleschuk ${ }^{2}$, M. Popesku ${ }^{13}$, E. Popova ${ }^{1}$, A. Porelli, E. Postnikov ${ }^{1}$, V. Prosin' ${ }^{1}$, V. Ptuskin 7 , A. Pushnin ${ }^{2}$, R. Raikin ${ }^{15}$, G. Rubtsov ${ }^{3}$, E. Ryabov ${ }^{2}$, Y. Sagan ${ }^{10,14}$, V. Samoliga ${ }^{2}$, B. Sabirov ${ }^{10}$, A. Silaev ${ }^{1}$, A. Silaev Jr. ${ }^{1}$, A. Sidorenkov ${ }^{3}$, A. Skurikhin ${ }^{1}$, V. Slunecka ${ }^{10}$, A. Sokolov ${ }^{11,12}$, Y. Suvorkin ${ }^{2}$, L. Sveshnikova ${ }^{1}$, V. Tabolenko ${ }^{2}$, B. Tarashchansky' ${ }^{2}$, L. Tkachev ${ }^{10,14}$, M. Tluczykont ${ }^{6}$, A. Tonaev ${ }^{2}$, N. Ushakov ${ }^{3}$, A.Vaidyanathan ${ }^{11}$, P. Volchugov ${ }^{1}$, D. Voronin ${ }^{3}$, R. Wischnewski ${ }^{8,10}$, A. Zagorodnikov ${ }^{2}$, D. Zhurov ${ }^{2,16}$, I. Yashin ${ }^{9}$

${ }^{1}$ Skobeltsyn Institute of Nuclear Physics MSU, Moscow, Russia, ${ }^{2}$ Institute of Applied Physics ISU, Irkutsk, Russia, ${ }^{3}$ Institute for Nuclear Research RAS, Moscow, Russia, ${ }^{4}$ Dipartimento di Fisica Generale Universiteta di Torino and INFN, Torino, Italy, ${ }^{5}$ Max-Planck-Institute for Physics, Munich, Germany, ${ }^{6}$ Institute for Experimental Physics, University of Hamburg, Germany, ${ }^{7}$ IZMIRAN, Moscow, Russia, ${ }^{8}$ DESY, Zeuthen, Germany, ${ }^{9}$ National Research Nuclear University MEPhI (Moscow Engineering Physics Institute), Moscow, Russia, ${ }^{10}$ Joint Institute for Nuclear Research, Dubna, Russia, ${ }^{11}$ Novosibirsk State University, NSU, Novosibirsk, Russia, ${ }^{12}$ Budker Institute of Nuclear Physics SB RAS, Novosibirsk, Russia, ${ }^{13}$ ISS, Bucharest, Romania, ${ }^{14}$ Dubna State University, Dubna, Russia, ${ }^{15}$ Altai State University, Barnaul, Russia, ${ }^{16}$ Irkutsk National Research Technical University, Irkutsk, Russia E-mail: nima.Iubsandorzhiev@yandex.ru

The hybrid installation TAIGA (Tunka Advanced Instrument for cosmic rays and Gamma Astronomy) is under construction to study cosmic and gamma rays at energies $10^{13}-10^{18} \mathrm{eV}$. It will incorporate timing wide-angle EAS Cherenkov array (TAIGA-HiSCORE) and number of imaging atmospheric Cherenkov telescopes (TAIGA-IACT). The first stage of TAIGA will include 100 wide-angle non-imaging optical stations of TAIGA-HiSCORE, distributed over an area of $\sim 1 \mathrm{~km}^{2}$ and three imaging telescopes at a distance of 300-500 m from each other. A combined method will be used to distinguish gamma-rays namely EAS parameters will be reconstructed by TAIGA-HiSCORE and a gamma-hadron separation will be carried out by TAIGA-IACT. With such an approach a hybrid detector will be built with a large area but with a relatively small number of telescopes.

36th International Cosmic Ray Conference -ICRC2019-

July 24th - August 1st, 2019

Madison, WI, U.S.A. 


\section{Introduction}

In the Tunka Valley close to Lake Baikal, the TAIGA observatory is under construction to study gamma and cosmic rays in the energy range of $10^{13}-10^{15} \mathrm{eV}$. It will be composed of the TAIGA-HiSCORE array of $\sim 100$ non-imaging wide-angle atmospheric Cherenkov stations covering an area of $\sim 1 \mathrm{~km}^{2}$ and the TAIGA-IACT array of 3 imaging atmospheric Cherenkov telescopes at distances of 300-500 m from each other. A hybrid detection of gamma-rays will be used, in which EAS parameters will be well reconstructed by the TAIGA-HiSCORE timing array and gamma-hadron separation will be more effectively provided by the TAIGA-IACT imaging telescopes [1-4]. The cosmic ray background suppression is $\sim 100$ at the energies above $80 \mathrm{TeV}$. The telescopes will operate in non-stereoscopic mode. Such an approach allows creating large area installation with a relatively small number of telescopes.

The TAIGA installation is sensitive to detect gamma-rays at energies above $30 \mathrm{TeV}$. One of the main objectives of the experiment is to search for Galactic sources of cosmic rays in the energy region near the knee of the cosmic ray spectrum. Expected integral sensitivity of the installation for 300 hours of source observation ( 2-3 operation seasons) in the energy range of $30-200 \mathrm{TeV}$ is $\sim 5 \cdot 10^{-13} \mathrm{TeV} \cdot \mathrm{cm}^{-2} \cdot \mathrm{sec}^{-1}$.

Furthermore, the TAIGA-MUON array of scintillation muon detectors [5] and a number of Schmidt-telescopes with a relatively wide FoV [15] will complement the installation.

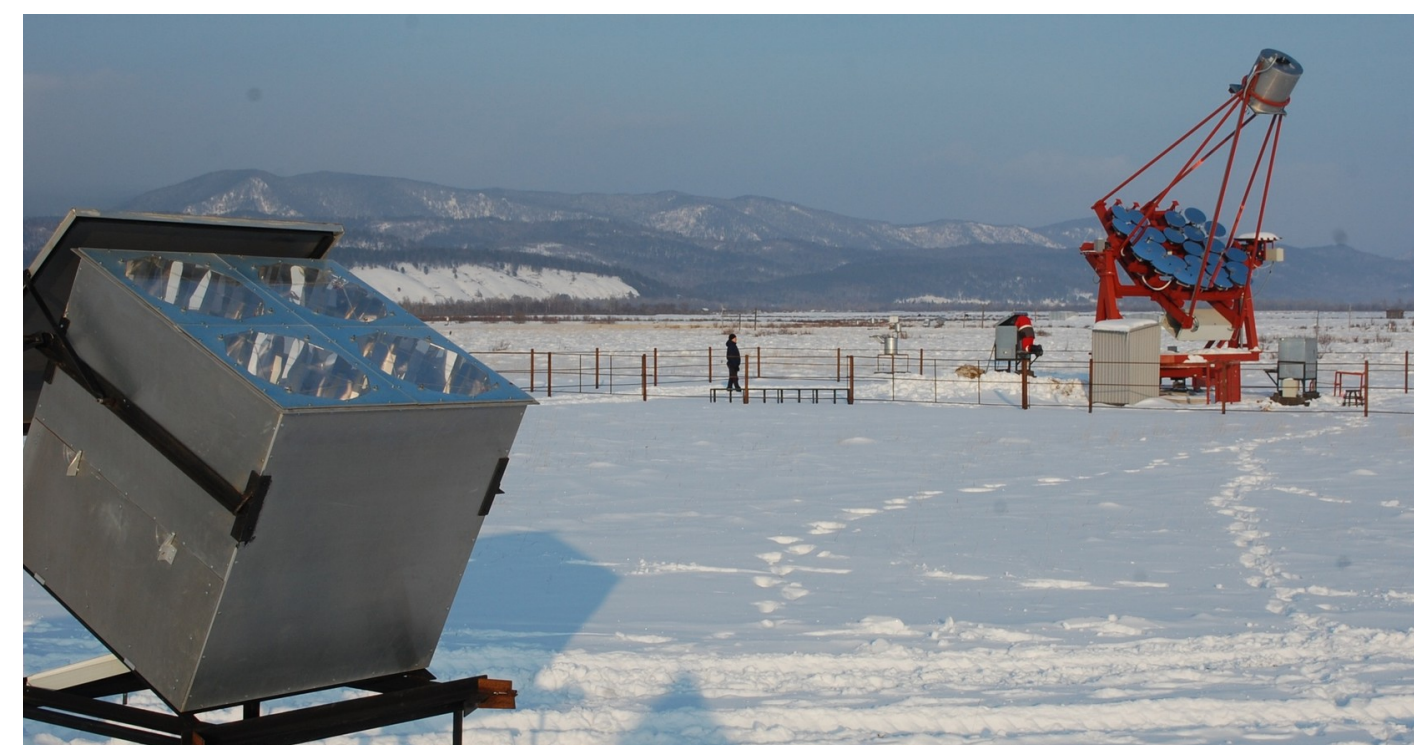

Fig. 1. The imaging telescope of the TAIGA-IACT array and the optical station of the TAIGA-HiSCORE array

\section{TAIGA-HiSCORE}

The TAIGA-HiSCORE array will be composed of 100 non-imaging wide-angle atmospheric Cherenkov stations distributed over an area of $\sim 1 \mathrm{~km}^{2}$ with an interstation spacing of $106 \mathrm{~m}$ [6-9]. Presently, 54 optical stations have been commissioned covering an area of $\sim 0.5$ $\mathrm{km}^{2}$. All optical stations are tilted by $25^{\circ}$ to the South to increase an observation time of the Crab Nebula. 
Each optical station is equipped by 4 eight-inch PMTs (R5912 or ET9352KB) complemented by Winston cones (ALANOD 4300UP). The total light sensitive area of station is $\sim 0.5 \mathrm{~m}^{2}$. Its $\mathrm{FoV}$ is $\sim 30^{\circ}(\sim 0.6 \mathrm{sr})$. The PMTs operate at a gain of $\sim 10^{4}$ under the night sky background light. Typical anode currents are $\sim 80 \mu \mathrm{A}$. Both anode and fifth dynode signals pass through fast preamplifiers with different gain factors to increase dynamic range. The amplified signals are sent via coaxial cables to readout electronics unit located a few meters from the station. The readout electronics consists of analog summator and ADC board based on the DRS4 chip. Analog summation of the signals of 4 PMTs allows reducing the energy threshold of the station by 2 times. If the amplitude of the analog sum exceeds 200 p.e., the ADC board produces trigger signal and digitizes input signals with a resolution of 14 bits, a sampling rate of $2 \mathrm{GHz}$ and a readout window of $500 \mathrm{~ns}$. The data is transmitted via optical fiber to the DAQ center. The counting rate of a single station is $10-15 \mathrm{~Hz}$. All optical stations are timesynchronized by two parallel systems, receiving the same front-end trigger signals: a custom system using a $100 \mathrm{MHz}$ clock distributed via optical fibers, and a White-Rabbit system.

Time calibration of the TAIGA-HiSCORE array is carried out several times per year. To do this, a powerful nanosecond LED-based light source $[10,11]$ is installed on a height at some distance from the array and illuminates the optical stations. This method determines the time delays between stations. The jitter of the measuring channel is less than $0.5 \mathrm{~ns}$.

The peak energy for hadrons in the differential energy spectrum measured by the TAIGAHiSCORE array is $\sim 80-100 \mathrm{TeV}$. For gamma-rays it is estimated to be $\sim 40-50 \mathrm{TeV}$. The angular resolution of the TAIGA-HiSCORE array achieves $\sim 0.1^{\circ}$ for big events with the number of triggered stations more than 10 and $\sim 0.4-0.5^{\circ}$ for events with more than 4 triggered stations. The preliminary integral cosmic ray energy spectrum measured by the TAIGA-HiSCORE array compared with other experiments is shown in fig. 2.

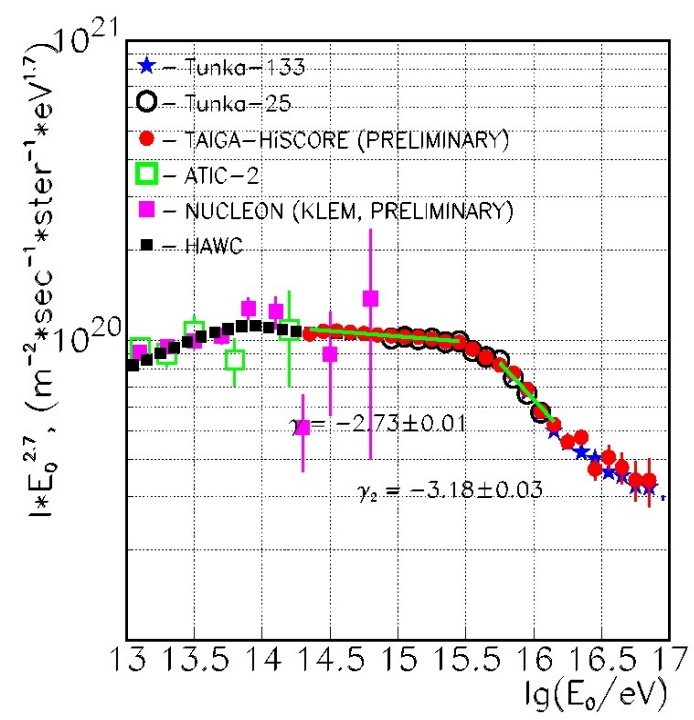

Fig. 2. Integral energy spectrum of primary cosmic rays measured by the TAIGA-HiSCORE array in comparison with other experiments 


\section{TAIGA-IACT}

TAIGA-IACT will be composed of 3 imaging atmospheric Cherenkov telescopes. The first telescope of TAIGA-IACT was put into operation in autumn 2017. Commissioning of the second telescope is planned this autumn. The imaging telescope is made by the Davies-Cotton design and has a spherical composite reflector with a diameter of $4.3 \mathrm{~m}$. The reflector is assembled from 34 segments. Its focal length is $4.75 \mathrm{~m}$.

The imaging camera of the first telescope is equipped with 560 hexagonally packed PMTs supplemented by Winston cones. PMT of XP1911 type with a diameter of $19 \mathrm{~mm}$ is used. The angular size of pixel is $0.36^{\circ}$. The total FoV of the camera is $9.6^{\circ}$.

The camera is divided into clusters. Each cluster consists of 28 PMTs, 4 HV divider boards, $4 \mathrm{HV}$ power supplies and readout electronics board. There are several outer clusters with the smaller number of PMTs. PMTs operate with the gain of $10^{4}$. The average anode current is $\sim 1 \mu \mathrm{A}$. The readout electronics of clusters is based on 64-channel chip ASIC MAROC3 [12]. It provides signal processing and cluster trigger signals, as well as monitoring of PMT anode currents and count rates. Each MAROC channel has preamplifier with adjustable gain factor, discriminator with adjustable threshold, integrating amplifier and 12-bit Wilkinson ADC. The signal from each PMT is split in parallel into 2 MAROC channels with different gain factors of preamplifiers to extend the dynamic range up to $~ 3000$ p.e.

To produce the cluster trigger it requires at least 3 (optionally 4 or 2) channels with signal amplitudes above 10 p.e. within 15 ns. Only channels with a high gain factor of preamplifier participate in the trigger. All clusters are connected to the central control board, which produces the main camera trigger, sends data and synchronizes time with the DAQ center via optical fibers. The triggered cluster and any additional cluster having at least one channel with a signal above 10 p.e. within a time window of 160 ns participate in the camera trigger.

For calibration of the camera, a nanosecond LED-based light source [19] is used. The light source is fixed in the center of the reflector and almost uniformly illuminates the whole camera. There are 2 PMTs with premeasured quantum efficiency in the camera. The sensitivity of remaining PMTs is evaluated using the excess noise factor method and compared with 2 calibrated PMTs.

The CCD-camera Prosilica GC1380 is installed at $1 \mathrm{~m}$ from the center of reflector and used for telescope pointing. It has a resolution of $1360 \times 1024$ pixels and a FoV of $31.4^{\circ} \times 23.6^{\circ}$. Each axis of the telescope is equipped with Phytron hybrid stepper motor, 17-bit shaft encoder and end-of-zone switches connected to the PhyMOTION control unit. Telescope pointing accuracy reaches $0.02^{\circ}[13,16]$. The telescope tracks the Crab Nebula and Mrk 421.

An example of a joint hadron-like event [14], while the telescope was tracking the Crab Nebula, is shown in fig. 3. In the figure EAS core position is projected on the camera plane as asterisk with additional scale factor: Rp $(\mathrm{cm}) / \mathrm{Rc}(\mathrm{cm})=1500$, where $\mathrm{Rp}$ is the distance from the telescope to EAS core position and Rc is the distance from the camera center to the asterisk. For events coming from the source to which the telescope is oriented the line connecting the projection of the EAS axis and the center of gravity of the image should cross the camera center. Within coincidence window of $\sim 2 \mu$ s the same event was detected by 15 stations of the TAIGAHiSCORE array with following EAS parameters: $\mathrm{E}=840 \mathrm{TeV}, \theta=30.1^{\circ}, \varphi=33.6^{\circ}, \mathrm{Rp}=134$ $\mathrm{m}$, the angle between the direction of the shower and direction of the source is $\psi=0.47^{\circ}$. 


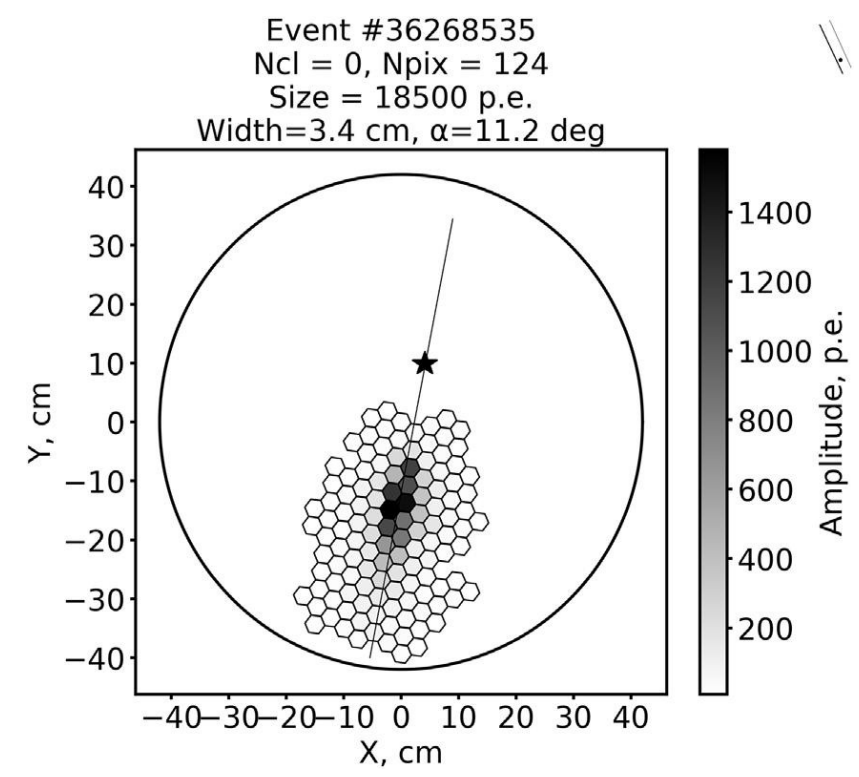

Fig. 3. An example of a joint hadron-like event with the projection of EAS core position (asterisk) on the camera plane

\section{Smidt-telescope with a large FoV on SiPMs}

To select events from gamma-rays against the background of EAS from cosmic rays, data from the TAIGA-HiSCORE array are combined with data on the image from IACT. Such a hybrid approach is effective for selection of events induced by gamma-rays. A disadvantage of the existing hybrid installation is a significant difference in the apertures of the TAIGAHiSCORE array and the Cherenkov telescopes. With camera FoV of $10^{\circ}$, solid angle of IACT is 25 times smaller than solid angle of the TAIGA-HiSCORE array and accordingly only $4 \%$ of events detected by the TAIGA-HiSCORE array fall into IACT FoV. To study energy range above $30 \mathrm{TeV}$ one can use a wide-angle camera with a FoV of $15-20^{\circ}$ and an effective recording area of no more than $1 \mathrm{~m}^{2}$, which will allow observing at least 5-7 sources during the night. The energy threshold of the camera will be approximately $10 \mathrm{TeV}$. Percentage of joint events with the TAIGA-HiSCORE array will increase by 2-3 times. In the future diameter of FoV could be increased to $60^{\circ}$ and become very comparable with the aperture of the TAIGA-HiSCORE array.

The optical system of the telescope can be designed by using spherical mirrors or using a system of Fresnel lenses, or a combination of the two above mentioned technologies. The silicon photomultipliers (SiPMs) will be used as light sensors in the imaging camera with the total number of pixel of $\sim 1200$.

\section{Conclusion}

The TAIGA observatory is designed to study gamma and cosmic rays at the energy range of $10^{13}-10^{18} \mathrm{TeV}$. At the first stage, the observatory will include the array of $\sim 100$ non-imaging wide-angle atmospheric Cherenkov stations covering a total area of $\sim 1 \mathrm{~km}^{2}$ and 3 imaging atmospheric Cherenkov telescopes at a distance of 300-500 m from each other. The observatory 
will also be complemented by muon scintillator detectors with a total area of $200 \mathrm{~m}^{2}$ and Schmidt telescopes with wide FoV of 15-20 degrees. It is planned to complete construction of the first stage of the TAIGA observatory in 2020-2021.

We are preparing for operating the SST-1M IACT Camera [17], originally developed for the CTA project, at the TAIGA site. After adapting to Siberian environmental conditions, this will permit for useful cross calibration and for full event time reconstruction by recording the waveform for each pixel.

In future, it is planned to develop the observatory up to $10 \mathrm{~km}^{2}$ with the array of $\sim 1000$ wide-angle optical stations, $~ 16$ IACTs, muon scintillator detectors with a total area of $2000 \mathrm{~m}^{2}$ and number of Schmidt telescopes.

\section{Acknolegments}

This work was supported by the Russian Foundation for Basic Research, grant 16-2913035, and the Russian Science Foundation (grant 19-72-20230, Section 4), grant 19-72-20067 of the Russian Science Foundation (Section 3), the Russian Foundation for Basic research (grant 18-32-00460), the Russian Federation Ministry of Education and Science (agreem.: 3.9678.2017/8.9, 3.904.2017/4.6, 3.6787.2017/7.8, 3.6790.2017/7.8, 3.5917.2017/Bch, 19-7220173) and by the European Union’s Horizon 2020 programme (Grant No 653477).

\section{References}

[1] N.Budnev et al (TAIGA Collaboration), TAIGA experiment: present status and perspectives, JINST 12 (2017) no. 08, C08018

[2] L. Kuzmichev et al (TAIGA Collaboration), Cherenkov EAS arrays in the Tunka astrophysical center: From Tunka-133 to the TAIGA gamma and cosmic ray hybrid detector, NIM A (in press), https://doi.org/10.1016/j.nima.2019.01.056

[3] L. Kuzmichev. et al (TAIGA Collaboration), Tunka Advanced Instrument for cosmic rays and Gamma Astronomy (TAIGA): Status, results and perspectives, EPJWeb Conf. v. 145 (2017) 01001

[4] E. Postnikov et al, Commissioning the joint operation of the wide angle timing HiSCORE Cherenkov array with the first IACT of the TAIGA experiment, PoS (ICRC2017) 756

[5] I. Astapov et al (TAIGA Collaboration), Scintillation detectors for the TAIGA experiment, NIM A 936 (2019) 254-256

[6] M. Tluczykont et al, The ground-based large-area wide-angle gamma-ray and cosmic-ray experiment HiSCORE, Adv.Space Res. 48, 1935-1941 (2011)

[7] M. Tluczykont et al, The HiSCORE concept for gamma-ray and cosmic-ray astrophysics beyond $10 \mathrm{TeV}$, Astropaticle Phys. 56, 42 (2014)

[8] M.Tluczykont et al (TAIGA Collaboration), Proceedings of RICAP16 6th Roma International Conference on Astroparticle Physics. EPJ Web Conferences

[9] O. Gress et al, The wide-aperture gamma-ray telescope TAIGA-HiSCORE in the Tunka Valley: Design, composition and commissioning, NIM A 845 (2017) 367 
[10] B.K.Lubsandorzhiev et al. LED based powerful nanosecond light sources for calibration systems of deep underwater neutrino telescopes, Nucl. Instrum. and Meth. A. 2009. V.602. P.220223.

[11] B.K. Lubsandorzhiev et al. Calibration system of the TUNKA-133 EAS Cherenkov Array, Proc. of the 32nd ICRC. Beijing China. August 11-19, 2011. ID-1048. Vol.3. P.243.

[12] I. Yashin et al, Imaging camera and hardware of Tunka-IACT, PoS (ICRC2015) 986

[13] D. Zhurov et al, First results of the tracking system calibration of the TAIGA-IACT telescope, J. Phys. Conf. Series 1181 (2019) 012045

[14] L.Sveshnikova et al (TAIGA Collaboration), Report at 26-th ECRS, Barnaul, 2018. To be pulished in Bull. Russ. Acad. Sci. Phys. 2019

[15] R. Mirzoyan, M.I. Andersen, A $15^{\circ}$ wide field of view imaging air Cherenkov telescope, Astroparticle Physics 31 (2009) 1-5

[16] N. Budnev et al (TAIGA-Collaboration), TAIGA - A hybrid array for high-energy gamma astronomy and cosmic-ray physics, Nuclear Instr. and Methods in Physics Research, A (2019), https://doi.org/10.1016/j.nima.2019.04.067 (Article in press)

[17] M.Heller et al. An innovative silicon photomultiplier digitizing camera for gamma-ray astronomy, Eur. Phys. J.C77 (2017) 1, 47, https://arxiv.org/pdf/1607.03412.pdf

[18] Y. Sagan et al (TAIGA collaboration), TAIGA observatory: IACT fabrication and tests, PoS (ICRC2019) 776

[19] B.K.Lubsandorzhiev et al. A LED Flasher for TUNKA EAS Experiment // Proc. of the 30th ICRC, R.Caballero, J.C.D’Olivo, G.Medina-Tanco, L.Nellen, F.A.Sanchez, J.F.Valdes-Galicia (eds.) Universidad nacional Autonoma de Mexico, Mexico City, Mexico, 2008. Merida Mexico, July 2007. Vol.5(He part 2). P.1117. / arXiv:0709.0458 [physics]. 